\title{
Desenvolvimento de Processos Fermentativos e Análise Sensorial no Ensino de Engenharia Química: Uma experiência no ensino remoto
}

DOI: 10.37702/2175-957X.COBENGE.2021.3663

Madrith Sthel Costa Duarte - madrith.duarte@prof.una.br

Centro Universitário Una

Rua Esmaltina 10

31015-110 - Belo Horizonte - MG

Margarete Aparecida Pereira - margarete.pereira@gmail.com

Centro universitário Una

Nancy de Vasconcelos Gomes 125

31030-070 - Belo Horizonte - MG

Resumo: o presente trabalho investigou a aplicação de práticas de ensino com o intuito de perceber a aceitação e aprendizagem dos estudantes. Foram utilizadas aulas formais expositivas referentes aos temas de bioquímica aplicada a fenômenos de fermentação e lançado o desafio aos estudantes de desenvolvimento de um processo fermentativo com recursos caseiros. Os processos selecionados foram: cerveja, cachaça, vinho, iogurte, queijo e pão. Os grupos de no máximo seis estudantes se reúnem remotamente e desenvolvem algum dos processos selecionados, criam POPs (procedimentos operacionais padrão) e elaboram um plano de aplicação em escala. As variáveis dos processos são investigadas pelos estudantes a fim de ampliar a formação do engenheiro tirando-os do lugar de conhecimentos fragmentados para uma formação por competências. Os processos realizados pelos estudantes em suas residências se mostrou eficaz do ponto de vista da aprendizagem assim como o estudo de análise sensorial que se mostrou eficaz com aplicação de produtos caseiros de baixo custo com substituição ao comparado com análises realizadas em laboratório com soluções adequadas.

Palavras-chave: Fermentação. Análise Sensorial. Ensino Remoto 


\section{DESENVOLVIMENTO DE PROCESSOS FERMENTATIVOS E ANÁLISE SENSORIAL NO ENSINO DE ENGENHARIA QUÍMICA: UMA EXPERIÊNCIA NO ENSINO REMOTO}

\section{INTRODUÇÃO}

O ensino de bioquímica para alunos de engenharia química é um ganho na formação profissional destes uma vez que um dos setores que demanda mão de obra qualificada é o setor de alimentos, onde tais conteúdos estão presentes. Contudo os conceitos de bioquímica estudados nas disciplinas formais são por vezes complexos e tediosos aos estudantes, sobretudo em tempos de ensino remoto. Com as novas demandas de ensino aprendizagem muitas adaptações foram necessárias a fim de manter alunos engajados com o processo de aprendizagem, e professores com metodologias de ensino que garanta acompanhamento adequado dos aprendizes.

O ensino para compreensão é uma metodologia que considera que entender é um domínio profundo e diretamente ligado com a prática. Os estudantes precisam ser capazes de fazer relações com diversos conhecimentos. Assim, o estudo de conceitos bioquímicos de forma isolada de uma prática profissional pode levar estudantes a um estudo esvaziado de significação e aplicação.

Alguns desafios precisam ser superados no que diz respeito a experimentação e inserção prática no mercado. Culturalmente os estudantes ainda esperam de disciplinas na formação universitária um grande volume de conteúdos de forma expositiva pelo professor o que causa a impressão de muito conhecimento. E o que todo estudante almeja é a compreensão. Outro anseio recorrente em acadêmicos é a oportunidade da experiência profissional em grandes indústrias o que pode ser um desafio para as instituições de ensino no que tange as relações comerciais com temas delicados como o segredo industrial.

Alinhados ao propósito de formação profissional com significados, foi proposto para a Unidade Curricular de Fenômenos Bioquímicos e suas Aplicações do componente curricular do curso de Engenharia Química para turmas de 5 período na cidade de Belo Horizonte o desenvolvimento de produtos alimentícios que levassem em consideração um processo fermentativo. Após a observação do processo, mesmo sendo realizado em residências de forma rudimentar, análise sensorial foi associada ao desenvolvimento bem como os POPs (procedimentos operacionais padrão) para o processo.

\section{DESENVOLVIMENTO}

\subsection{Considerações gerais}

Segundo Palermo (2015), a análise sensorial é uma técnica de análise de alimentos e outros materiais através de órgão sensoriais humanos. Os sistemas olfativo o, gustativo, tátil, auditivo e visual são os instrumentos para a avaliação dos atributos dos alimentos e de suas propriedades sensoriais por meio de técnicas e métodos adequados, que garantam a qualidade dos resultados (PALERMO, 2015; TEIXEIRA; 2009). Esquematicamente, definição da análise sensorial pode ser representadas na figura 1, como sugerido por Esteves (2014). 
Figura 1 - llustração do conceito de análise sensorial

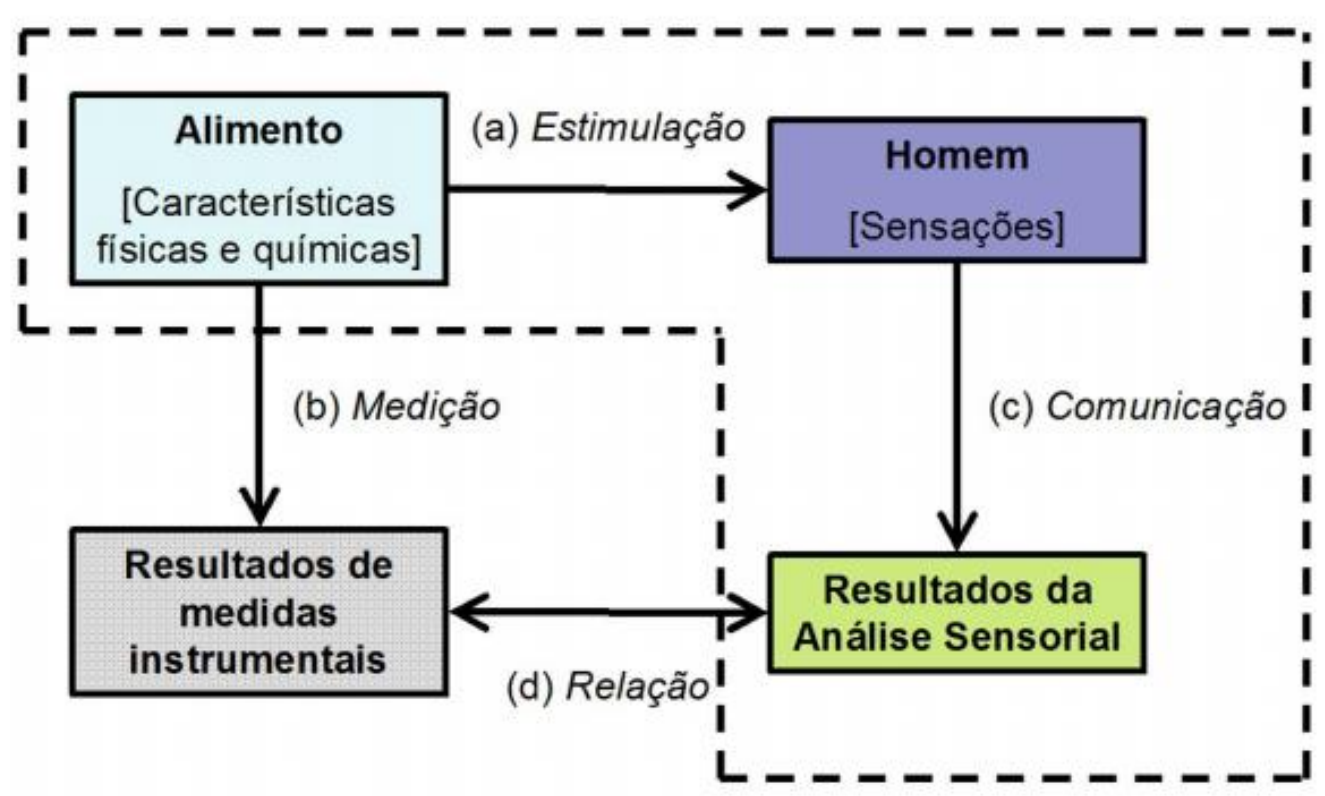

Fonte: ESTEVES, 2014

\section{$2.2 \quad$ Testes sensoriais}

Os aromas sempre atraíram a atenção da humanidade. Existem registros tratados sobre aromas elaborados pelos gregos em 300 a.C. Na Europa, a degustação era usada para controlar a qualidade em cervejarias e destilarias. Como disciplina, a análise sensorial surgiu na década de 1940 em países Escandinavos e nos Estados Unidos (ESTEVES, 2014; NESPOLO et al, 2015; TEXEIRA, 2009). Técnicas de avalição passaram a ser desenvolvidas para obtenção de produtos agradáveis ao paladar dos consumidores.

A aceitação dos alimentos por consumidores, está relacionada com a percepção sensorial dos mesmos, e é comum que alimentos com altamente nutritivos podem não ter boa aceitação de dos consumidores. Técnicas de análise sensorial é um disciplina multidisciplinar, sendo respaldada pela estatística por análises estatísticas e validação de resultados e da psicologia (OLIVAS - GASTÉLUM et al., 2009).

A avaliação sensorial dos alimentos na indústria pode ser realizada por meio de diferentes testes dependendo do tipo de informação que se deseja obter. Segundo OlivasGastélum et al. (2009) podem há três tipos principais de testes: teste afetivos, teste de discriminação e testes descritivos.

Ainda de acordo com os autores supracitados, o teste afetivo é aquele que se busca avaliar a aceitação do produto a partir da reação do avaliador. O teste de discriminação deseja-se avaliar se duas amostras são suficientemente diferentes para ser catalogadas como tal. Finalmente, as provas descritivas definem as propriedades de um alimento e medi-las de maneiras mais objetivas possíveis. Para que os testes sensoriais obtenham os resultados desejados é importante o treinamento dos avaliadores para determinar a incapacidade sensoriais, como detecção de cor, de odor e de gostos básicos, a exatidão sensorial e a avaliação da percepção sensorial. Neste trabalho, foi aplicado o teste de discriminação. Optou-se por este tipo de teste, visto que, o mesmo é amplamente utilizado na academia e na indústria para o controle de qualidade, para o estudo de impactos por 
trocas na formulação e no processo, assim como a habilidade dos consumidores de diferenciar entre produtos semelhantes.

\subsection{Análise sensorial como ferramenta de qualidade}

A análise sensorial vem sendo aplicada no desenvolvimento e melhoramento de produtos, controle de qualidade, estudos sobre armazenamento e desenvolvimento de processos (LANZILLOTTI e LANZILLOTTI, 1999).

A norma NBR 12806 de 1993 define qualidade como o conjunto de características que diferencia unidades individuais de um produto. Segundo Palermo (2015), as características dos alimentos tendem a ser percebidas na seguinte ordem:

- Aparência: cor, tamanho e forma, textura da superfície, translucidez, grau de carbonatação.

- Odor e aroma: o odor é detectado quando os voláteis de um produto entram na cavidade nasal são percebidas pelo sistema olfatório; o aroma é percebido pela região retronasal quando o alimento é colocado na boca, consistência e textura, sabor.

- Consistência e textura: viscosidade é atribuída para líquidos newtonianos homogêneos; a consistência é para líquidos semissólidos não newtonianos e heterogêneos; a textura, para sólidos ou semissólidos.

- Sabor (aromático, odor químico, gosto): aromáticos são voláteis percebidos pelo sistema olfatório causados por substâncias na boca, via retronasal; odores químicos são estimulantes neurais das membranas das cavidades nasal e bucal que provocam sensação de adstringência, apimentado, gelado, amargo, sabor metálico etc; o gosto e percepção gustativa (salgado, ácido, doce, amargo e umami) causada pelas substâncias solúveis na boca.

Em programas de controle de qualidade, esta interação tem sido usada para medir a qualidade do alimento (LANZILLOTTI e LANZILLOTTI, 1999).

\section{METODOLOGIA}

O trabalho foi desenvolvido em duas turmas de alunos com dois encontros semanais em aulas com 150 minutos de duração. Duas etapas foram realizadas: exposição dialogada sobre os temas processos fermentativos, micro-organismos, proteínas e processos enzimáticos; em segunda etapa os alunos escolheram um produto alimentício para executar e práticas para o treinamento de análise sensorial foram desenvolvidas.

Os processos selecionados pelos alunos foram a produção de cerveja, vinho, cachaça, queijo, hidromel, kombucha, iogurte, leite fermentado e pães. Cada grupo composto de no máximo seis integrantes se reuniram de forma remota para alinhar seus processos e executá-los em suas residências onde podem realizar análise sensorial com o grupo restrito a seus familiares como forma de evitar contatos com a possível proliferação do Corona vírus causador da Covid-19.

Um dos desafios para a realização da análise sensorial é o treinamento do analisador. Para reconhecimento de gostos primários e suas misturas foram realizadas soluções com ingredientes alternativos aos propostos para análise. A tabela 1 foi apresentada aos estudantes para reconhecimento de reagentes e suas concentrações quanto aos gostos primários de intensidade fraca, moderada e forte. 
Tabela 1 - Quantidades para o preparo de $500 \mathrm{ml}$ de soluções para reconhecimento de gostos primários por analisadores sensoriais.

\begin{tabular}{c|c|c|c|c|c|c|c}
\multicolumn{2}{c|}{ Gosto Doce } & \multicolumn{2}{c|}{ Gosto Ácido } & \multicolumn{2}{c|}{ Gosto Salgado } & \multicolumn{2}{c}{ Gosto Amargo } \\
\hline Fraco & $2,0 \mathrm{~g}$ & Fraco & $0,10 \mathrm{~g}$ & Fraco & $0,5 \mathrm{~g}$ & Fraco & $0,2 \mathrm{~mL}$ \\
\hline Moderado & $4,0 \mathrm{~g}$ & Moderado & $0,15 \mathrm{~g}$ & Moderado & $1,0 \mathrm{~g}$ & Moderado & $0,5 \mathrm{~mL}$ \\
\hline Forte & $6,0 \mathrm{~g}$ & Forte & $0,20 \mathrm{~g}$ & Forte & $1,5 \mathrm{~g}$ & Forte & $1,0 \mathrm{~mL}$ \\
\hline
\end{tabular}

Fonte: Os autores

Como alternativa aos reagentes para o reconhecimento de gostos ácido e amargo os substituintes escolhidos foram o limão Taiti e água tônica.

A tabela 2 identifica as quantidades utilizadas para a substituição dos produtos padrão, e a tabela 3 as misturas de gostos primários.

Tabela 2 - Quantidades e medidas propostas como alternativa a soluções padrão de $500 \mathrm{~mL}$. Onde: $\mathrm{cc}=$ colher de chá; $\mathrm{cs}=$ colher de sopa, $\mathrm{m}=$ medida, $\mathrm{F}=$ frasco

\begin{tabular}{c|c|c|c|c|c|c|c}
\hline \multicolumn{2}{c|}{ Gosto Doce } & \multicolumn{2}{c|}{ Gosto Ácido } & \multicolumn{2}{c|}{ Gosto Salgado } & \multicolumn{2}{c}{ Gosto Amargo } \\
\hline Fraco & $\begin{array}{c}2,0 \mathrm{~g} \\
(1 \mathrm{cc})\end{array}$ & Fraco & $\begin{array}{c}0,10 \mathrm{~g} \\
(1 \mathrm{cc})\end{array}$ & Fraco & $\begin{array}{c}0,5 \mathrm{~g} \\
(1 / 3 \mathrm{cc})\end{array}$ & Fraco & $\begin{array}{c}0,2 \mathrm{~mL} \\
(1 / 2 \mathrm{~m}+ \\
1 / 2 \mathrm{água})\end{array}$ \\
\hline Moderado & $\begin{array}{c}4,0 \mathrm{~g} \\
(2 \mathrm{cc})\end{array}$ & Moderado & $\begin{array}{c}0,15 \mathrm{~g} \\
(1 \mathrm{cs})\end{array}$ & Moderado & $\begin{array}{c}1,0 \mathrm{~g} \\
(2 / 3 \mathrm{cc})\end{array}$ & Moderado & $\begin{array}{c}0,5 \mathrm{~mL} \\
(1 / 2 \mathrm{~F}+1 / 2 \\
\text { água })\end{array}$ \\
& & & & & & & \\
\hline Forte & $6,0 \mathrm{~g}$ & Forte & $\begin{array}{l}0,20 \mathrm{~g} \\
(2 \mathrm{cs})\end{array}$ & Forte & $1,5 \mathrm{~g}$ & Forte & $1,0 \mathrm{~mL}$ \\
& $(3 \mathrm{cc})$ & & & $(1 \mathrm{cc})$ & & $(1 \mathrm{~m})$ \\
\hline
\end{tabular}

Fonte: Os autores

Tabela 3 - Preparo de soluções de $500 \mathrm{ml}$ para misturas de gostos primários.

\begin{tabular}{c|c|c}
\hline Gostos primários & Substâncias & Quantidades $(500 \mathrm{~mL})$ \\
\hline Sal + ácido & $\mathrm{NaCl}+$ suco de limão & 9 \\
\hline Doce + ácido & Açúcar + suco de limão & $2 \mathrm{cs}+1 \mathrm{cs}$ \\
\hline Ácido + amargo & Suco de limão + água tônica & $1 \mathrm{cs}+100 \mathrm{~mL}$ \\
\hline Sal + amargo & $\mathrm{NaCl}+$ água tônica & $1 \mathrm{cs}+200 \mathrm{~mL}$ \\
\hline Sal + doce & $\mathrm{NaCl}+$ açúcar & $1 \mathrm{cs}+1 \mathrm{cs}$ \\
\hline Doce + amargo & Açúcar + água tônica & $1 \mathrm{e}^{1 / 2} \mathrm{cs}+150 \mathrm{~mL}$
\end{tabular}

Fonte: Os autores

Durante o registro dos testes realizados, os estudantes deveriam registrar o gênero, a idade e se o participante havia contraído covid-19, para posterior análise de sensibilidade de sensorial de acordo com estes parâmetros. Os dados foram tabulados pelos estudantes para que os perfis de reconhecimento de sabor de seus analisadores fossem reconhecidos. A partir do número de acertos de cada sabor, o threshold que é o limiar de sensibilidade ao sabor, foi identificado.

\section{APRESENTAÇÃO E DISCUSSÃO DOS RESULTADOS}

A seleção dos analisadores foi realizada pelos alunos entre seus familiares. Destes, $68 \%$ são do sexo feminino e $32 \%$ do sexo masculino. De acordo com os resultados preliminares pode-se observar que, numericamente, pessoas do sexo masculino tem média maior que as do sexo feminino, como pode ser observado na tabela 5. Porém, ainda não é possível afirmar que tal fato será mantido.Para o reconhecimento de gostos primários 19 analisadores tabulados mostram que há uma boa percepção de sabores com uma média 
de 13 acertos entre as 18 amostras analisadas. A percepção é maior para os sabores ácido e doce entre o grupo de pessoas testadas como mostrado na tabela 4.

Tabela 4 - Relação de reconhecimento de sabores.

\begin{tabular}{c|c}
\hline Sabores & Sabores primários mais reconhecidos \\
\hline Ácido & 3 \\
\hline Ácido e doce & 1 \\
\hline Amargo & 1 \\
\hline Doce & 6 \\
\hline Doce e ácido & 1 \\
\hline Doce, Salgado e Ácido & 1 \\
\hline Sal & 2 \\
\hline Salgado & 1 \\
\hline Salgado e ácido & 1 \\
\hline Todos menos o ácido & 1 \\
\hline Todos menos o amargo & 1 \\
\hline Total Geral & 19
\end{tabular}

Fonte: Os autores

Tabela 5 - Média do número de acertos por gênero

\begin{tabular}{c|c}
\hline Feminino & Masculino \\
\hline 8,30 & 10,5 \\
\hline
\end{tabular}

Fonte: Os autores

Ao analisar-se a influência da covid-19, pode-se observar que a média de acertos dos avaliadores que contraíram o SARS-COV 2 é menor numericamente, como pode-se observar na tabela 6.

Tabela 6 - Média do número de acertos avaliadores que contraíram ou não SARS-COV2

\begin{tabular}{c|c}
\hline Não contraíram SARS-COV 2 & Contraíram SARS-COV 2 \\
\hline 9,2 & 8,7 \\
\hline
\end{tabular}

Fonte: Os autores

O método adotado pelos estudantes foram bem aceitos pelos avaliadores treinados. Ainda pode-se constatar que as soluções adaptadas para o momento de pandemia foram efetivas.

\section{CONSIDERAÇÕES FINAIS}

A pandemia trouxe desafios enormes para 0 ensino de engenharia. $O$ desenvolvimento de técnicas de produção e a aplicação de conceitos foram adaptados para que os alunos fossem protagonistas no processo de ensino-aprendizagem. A aplicação de conceitos de bioquímica por meio da produção de alimentos fermentados e do uso da 
análise sensorial, mostrou-se efetiva, pois percebeu o aumento da participação dos mesmos nas aulas síncronas e maior entendimento do conteúdo.

Os resultados preliminares mostram que os alunos são capazes de promover treinamentos e aplicar conteúdos e elaborar procedimentos operacionais padrão.

\section{REFERÊNCIAS}

ASSOCIAÇÃO BRASILEIRA DE NORMAS TÉCNICAS. NBR 12806:Análise sensorial dos alimentos e bebidas. Rio de Janeiro, 1993.

ESTEVES, Eduardo. Introdução à Análise Sensorial. Instituto Superior de Engenharia da Universidade do Algarve, Departamento de Engenharia Alimentar, Faro. Disponível em http://w3.ualg.pt/ eesteves>. Acesso em: 05 de maio de 2021.

LANZILLOTTI, Regina Serrão; LANZILLOTTI, Haydée Serrão. Análise sensorial sob o enfoque da decisão fuzzy. Rev. Nutr., Campinas, v. 12, n. 2, p. 145-157, Aug. 1999.

NESPOLO, Cássia Regina et al. Práticas em Tecnologia de Alimentos. 1 ed. Porto Alegre: Editora Artmed. 2015.

OLIVAS - GASTÉLIUM, R.; MOORILLON, G. V; GASTÉLIUM-FRANCO, M.G. Las pruebas de diferencia en el análisis sensorial de los alimentos. Tecnociencia Chihuahua, Chihuahua, v.3, n. 1, p. 1-7

PALERMO, Jane Rizzo. Análise Sensorial: Fundamentos e Técnicas. 1 ed. Rio de Janeiro: Editora Atheneu . 2015.

TEIXEIRA, L. V. Análise sensorial na indústria. Revista do Instituto de Latícinios Cândido Tostes. Juiz de Fora, , v. 64, n. 366, 0. 12-21, 2009

\section{INSTRUCTIONS FOR PREPARATION AND SUBMISSION OF WORKS TO THE SCIENTIFIC COMMITTEE OF XLIX BRAZILIAN CONGRESS OF ENGINEERING EDUCATION - COBENGE 2021}

Resumo: o presente trabalho investigou a aplicação de práticas de ensino com o intuito de perceber a aceitação e aprendizagem dos estudantes. Foram utilizadas aulas formais expositivas referentes aos temas de bioquímica aplicada a fenômenos de fermentação e lançado o desafio aos estudantes de desenvolvimento de um processo fermentativo com recursos caseiros. Os processos selecionados foram: cerveja, cachaça, vinho, iogurte, queijo e pão. Os grupos de no máximo seis estudantes se reúnem remotamente e desenvolvem algum dos processos selecionados, criam POPs (procedimentos operacionais padrão) e elaboram um plano de aplicação em escala. As variáveis dos processos são investigadas pelos estudantes a fim de ampliar a formação do engenheiro tirando-os do lugar de conhecimentos fragmentados para uma formação por competências. 
Os processos realizados pelos estudantes em suas residências se mostraram eficaz do ponto de vista da aprendizagem assim como o estudo de análise sensorial que se mostrou eficaz com aplicação de produtos caseiros de baixo custo com substituição ao comparado com análises realizadas em laboratório com soluções adequadas.

Palavras-chave: Fermentação. Análise Sensorial. Ensino Remoto

\begin{abstract}
This document investigated the application of teaching practices in order to perceive students' acceptance and learning. Formal expository classes were used on the topics of biochemistry applied to fermentation phenomena and the challenge was presented to students to develop a fermentative process with homemade resources. The selected processes were: beer, cachaça, wine, yogurt, cheese and bread. Groups of a maximum of six students meet remotely and develop any of the selected processes, create POPs (standard operating procedures) and develop a scale application plan. The variables of the processes are investigated by the students in order to expand the training of the engineer taking them from the place of fragmented knowledge to a training by competences. The processes carried out by students in their homes proved to be effective from the point of view of learning, as well as the study of sensory analysis that proved effective with the application of low-cost homemade products with substitution when compared to analyzes carried out in the laboratory with suitable solutions. .
\end{abstract}

Keywords: Yeast, Sensory Analysis, Fermentation 